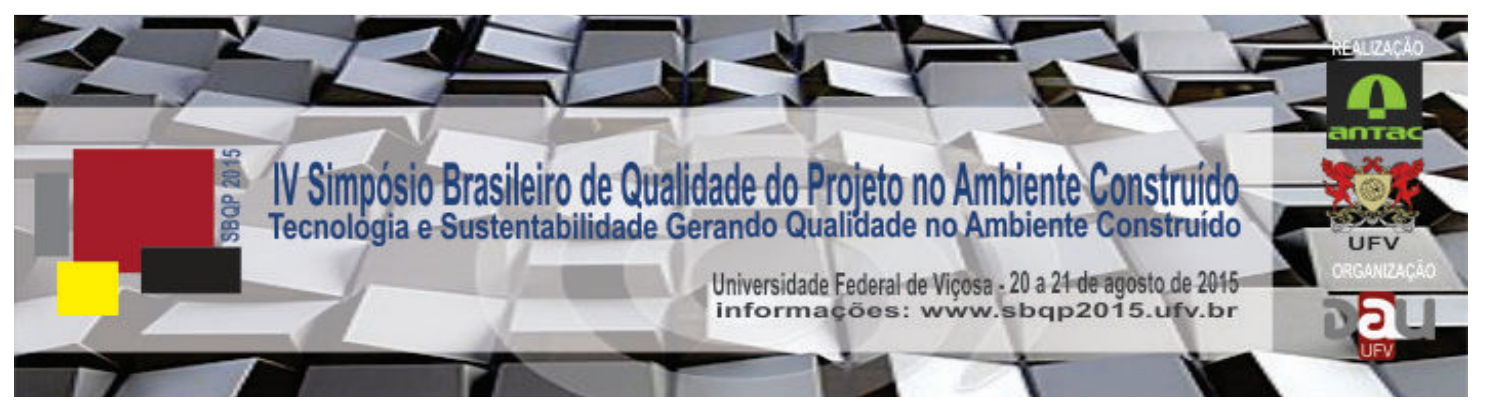

\title{
PROCESSO DE REGISTRO E MODELAGEM DE PREEXISTÊNCIAS COM USO DE ESCANER LASER TERRESTRE 1
}

\author{
SOUZA, Douglas L. \\ Universidade Federal de Viçosa, douglas@ufv.br \\ PINILLA, Sergio $M$. \\ Universidad del Tolima, smosquerap@ut.edu.co \\ TIBURCIO, Tulio M.S. \\ Universidade Federal de Viçosa, tiburcio@ufv.br \\ VALLEJO, Mario A. B. \\ Universidad del Tolima, andresvallejo@gmail.com \\ MILLAN, Luiza F. A. \\ Universidad del Tolima, Ifam1019@hotmail.com
}

\begin{abstract}
RESUMO
O desenvolvimento da tecnologia LiDAR no setor da construção civil tem ganhado novos potenciais com os atuais processos de projeto e registro da informação, sobretudo na sua interação com a plataforma BIM. Este artigo traz uma experiência de registro arquitetônico de uma edificação com uso de escâner laser terrestre e sua posterior modelagem a partir da nuvem de pontos. Como instrumento de análise foi elaborado uma comparação do processo de modelagem paramétrica com o método convencional, ambos a partir de dados escaneados pelo equipamento.
\end{abstract}

Palavras-chave: escâner laser, modelagem digital, nuvem de pontos, LiDAR.

\begin{abstract}
The development of LiDAR technology in the construction industry has gained new potential with the current design and recording of information processes, especially in its interaction with BIM plataform. This article brings an experience of architectural record of a building with use of terrestrial laser scanner and its subsequent modeling from the point cloud. As an analytical tool of this process, was drawn a comparison of parametric modeling process and the conventional method, both from scanned data by the equipment.
\end{abstract}

Keywords: laser scan, digital modeling, point clouds, LiDAR.

\section{INTRODUÇÃO}

\footnotetext{
1 Trabalho apresentado no IV SBQP 2015. Universidade Federal de Viçosa. Disponível em: doi> http://dx.doi.org/10.18540/2176-4549.6060
} 
O modelo geométrico de nuvem de pontos é o primeiro e mais comum produto gerado diretamente pelo escâner laser e pode ser usado para diversas finalidades, como medição, cadastro e simulação uma vez que estas nuvens podem ser importadas em software que recorrem a processos de modelagem para fins variados. A maioria das aplicações requerem a converção do modelo básico de nuvem de pontos em modelos mais complexos que podem ser parametrizados ou não.

Este modelo de nuvem de pontos, por sua natureza, características e estruturas de dados, representa um novo tipo de modelo geométrico tridimensional, diferente dos tradicionais modelos de arestas, de superfícies e de sólidos, utilizados até então por software de modelagem geométrica encontrados nas ferramentas CAD.

Com a implementação do escâner laser na construção civil, é possível a produção de modelos as-built rápidos e precisos da situação real de uma construção ou instalação, habilitando a realização de desenhos e modelos como a documentação ou manutenção da edificação baseados em dados reais evitando retrabalho e uso de informações inconsistentes. Além deste uso imediato, esta tecnologia permite comparar a evolução de uma edificação ou objeto construído ao longo do tempo usando diferentes escaneamentos que identifiquem deformações, movimentos e outras alterações que podem apresentar na superfície do objeto.

\section{FUNDAMENTAÇÃO}

A quantidade de informação mapeada pela tecnologia LiDAR (Light Detection and Ranging) amplia o campo de atuação dos profissionais que, usando a informação mapeada como suporte, têm como desenvolvem suas atividades.

A tecnologia LiDAR é um método de sensoriamento remoto que tem se desenvolvido em diversos setores, e ampliando a capacidade de armazenamento e precisão de seus equipamento são longo do tempo potencializando o uso do laser para mais diversas áreas. LICHTI, GORDON e STEWART (2002) já apresentaram estudo que orientava sobre os potenciais usos da tecnologia como instrumento para produção de informações as-built de construções e em alta resolução que permitem estudos de restauro de objetos e superfícies, registro de acidentes ou estudos forenses, entre outros, facilitados pelo grande volume de dados com alta precisão e definição minimizando a interferência humana sobre o local. De modo semelhante, KERSTEN, STERNBERG e MECHELKE (2009) se lançaram sobre o estudo do uso da tecnologia LiDAR como método para inspeção da construção e em comparação com desenhos dos projetos.

Com o desenvolvimento de outras ontologias, como o processo de modelagem BIM e a crescente demanda por precisão de dados, é necessária a exploração desta tecnologia para sua inserção nas atividades da indústria da construção.

Nesta pesquisa utilizamos plataformas de trabalho (software) com capacidade de trabalho BIM e de gestão de nuvens de pontos para compreender os limites 
que o processo de escaneamento e modelagem determinam no registro de edificações.

Mediante a implementação de plataformas de trabalho que tenham interação podemos ver a integração das tecnologias BIM e LiDAR em agências que tem desenvolvido este tipo de atividade, como a estadunidense GSA (General Services Administration) que em 2009 divulgou um programa de orientação sobre o uso de Laser Scanner 3D aliado a modelagem da informação da construção (GSA, 2009).

\section{MÉTODOS E RESULTADOS}

Esta pesquisa se lança sobre a comparação de dois processos de levantamento arquitetônico e a exploração do potencial da tecnologia laser para modelagem digital da construção. Como método para esta comparação, observamos que processo de coleta de dados é dependente da finalidade do projeto e sempre terá diversas etapas a seguir para consolidar uma informação clara, ordenada e precisa. De acordo com GROETELAARS e AMORIM (2011), as etapas geralmente utilizadas para um levantamento com tecnologia LiDAR terrestre são: 1) Planejamento; 2) Aquisição dos dados; 3) Pré-processamento; 4) Processamento; 5) Análise da precisão do modelo gerado e 6) Exportação dos produtos.

A partir desta sequência de etapas foi feito o levantamento de uma edificação de baixa complexidade e comparação com dois processos de modelagem com objetivo de análise de eficiência e precisão de informações resultantes de cada processo.

O método utilizado pela primeira equipe foi a modelagem tridimensional a partir de uma planta-baixa em formato DWG fruto de outro levantamento arquitetônico por uma terceira equipe que executou o desenho no ano anterior. A segunda equipe utilizou de um levantamento por escâner laser 3D e modelagem da geometria em software paramétrico. De posse dos dois modelos foi executado um comparativo que considerou tempo, exatidão das informações e recursos disponíveis para cada trabalho.

Como orientação ao processo utilizados a seguinte sequência de etapas proposta por GROETELAARS e AMORIN(2011):

\subsection{Fase 1 - Planejamento}

Como estudo comparativo, foi executado o escaneamento do edifício de pósgraduação do Departamento de Arquitetura e Urbanismo da Universidade Federal de Viçosa. A primeira etapa para coleta de dados deve ser o planejamento do levantamento porque tem importante papel no processo ao analisar o escopo do projeto de mapeamento e direcionando a quantidade de etapas e de informações a serem levantadas.

Como o equipamento opera com diferentes configurações de resolução, nos níveis mais altos o arquivo torna-se inviável para processamento devido à necessidade de uma máquina de grande capacidade de processamento para gerenciar toda a informação. Desta forma, considerou-se o menor número de posições que cobrisse toda a geometria a ser mapeada e com a menor perda de informação possível. Esta análise prévia da construção localizou pontos de 
maior complexidade ou de áreas que impediriam a visada pelo equipamento, definindo, portanto, um mapa do conjunto de posições de instalação do equipamento.

\subsection{Fase 2 - Preparo de marcadores}

A preparação da edificação ocorre a partir da fixação de alvos (figura 1 e 2) ou marcadores que identificam pontos estratégicos visíveis em pelo menos outra posição de locação do equipamento, de modo que, em duas posições distintas o mesmo alvo seja visto, reconhecido e possa ser usado para registro das duas cenas em uma. Ao todo foram locados vinte e dois alvos e isto deve-se à exigência do software de processamento desta informação que, neste caso, foi o Autodesk ReCap® que exige apenas três pontos em comum em cada cena.

\section{Figuras 1 e 2: Fixação dos alvos (esquerda) e posicionamento do laser ao redor da edificação.}
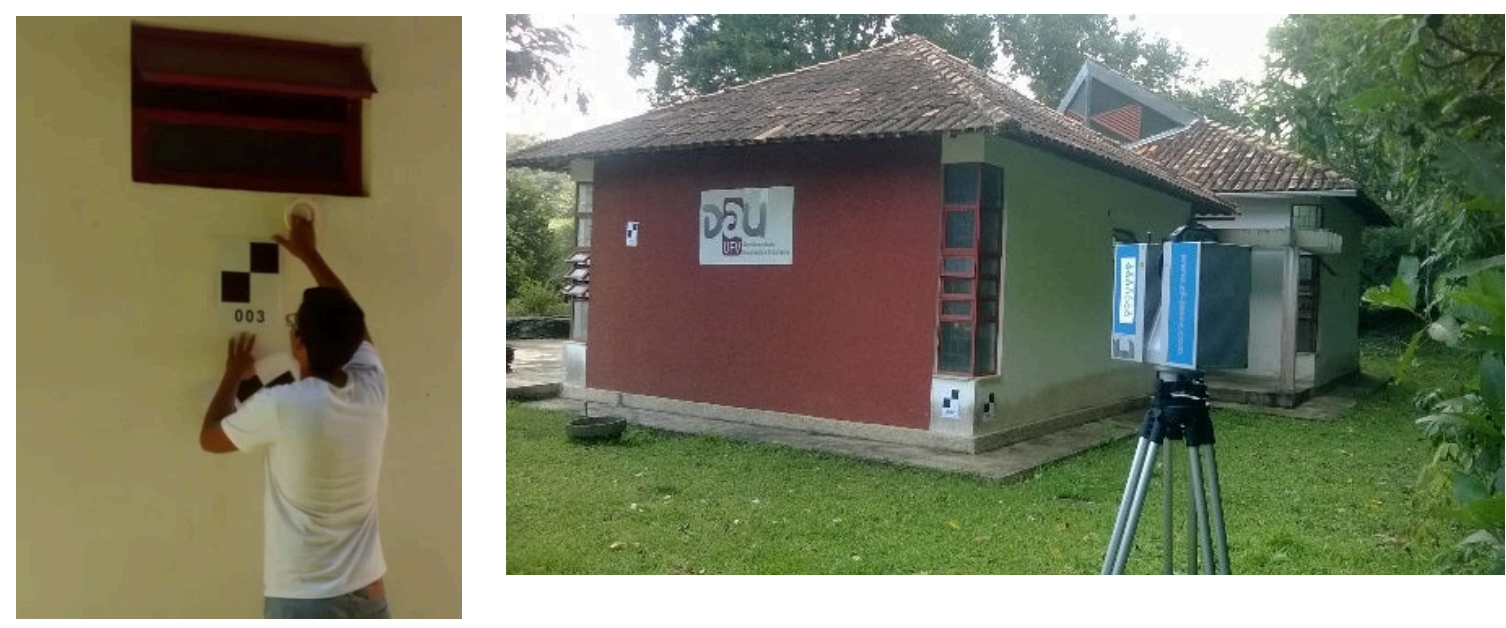

Fonte: resultados da pesquisa

\subsection{Fase 3 - Aquisição de dados por meio de escaneamento laser}

Para ajuste da configuração, da resolução ideal e do ângulo de registro, foram feitos testes antes do início do mapeamento o que demandou trinta minutos de trabalho até atingir o nível ideal para esta edificação, mas cada cena necessitou um tempo médio de instalação, nivelamento e escaneamento, totalizando dez minutos, sendo que o tempo útil, de trabalho efetivo do laser, foi de apenas três minutos na resolução escolhida.

Nesta fase adotamos uma configuração do equipamento que permitisse uma resolução que atendesse ao objetivo do projeto em mapear e criar um registro da edificação. O equipamento atinge a resolução máxima de $0,6 \mathrm{~mm}$ (a 10m do equipamento), mas, na área do projeto, esta resolução geraria arquivos que não poderiam ser processados em máquinas de baixa ou média capacidade de processamento. Para minimizar este problema determinamos um limite para 0 ângulo horizontal a fim de reduzir a área de mapeamento, arquivos maiores $e$ uma demanda maior de capacidade de processamento. 
Figura 3: Última posição de escaneamento executada ao final do dia (acima). Imagem da mesma posição em nuvem de pontos (abaixo).

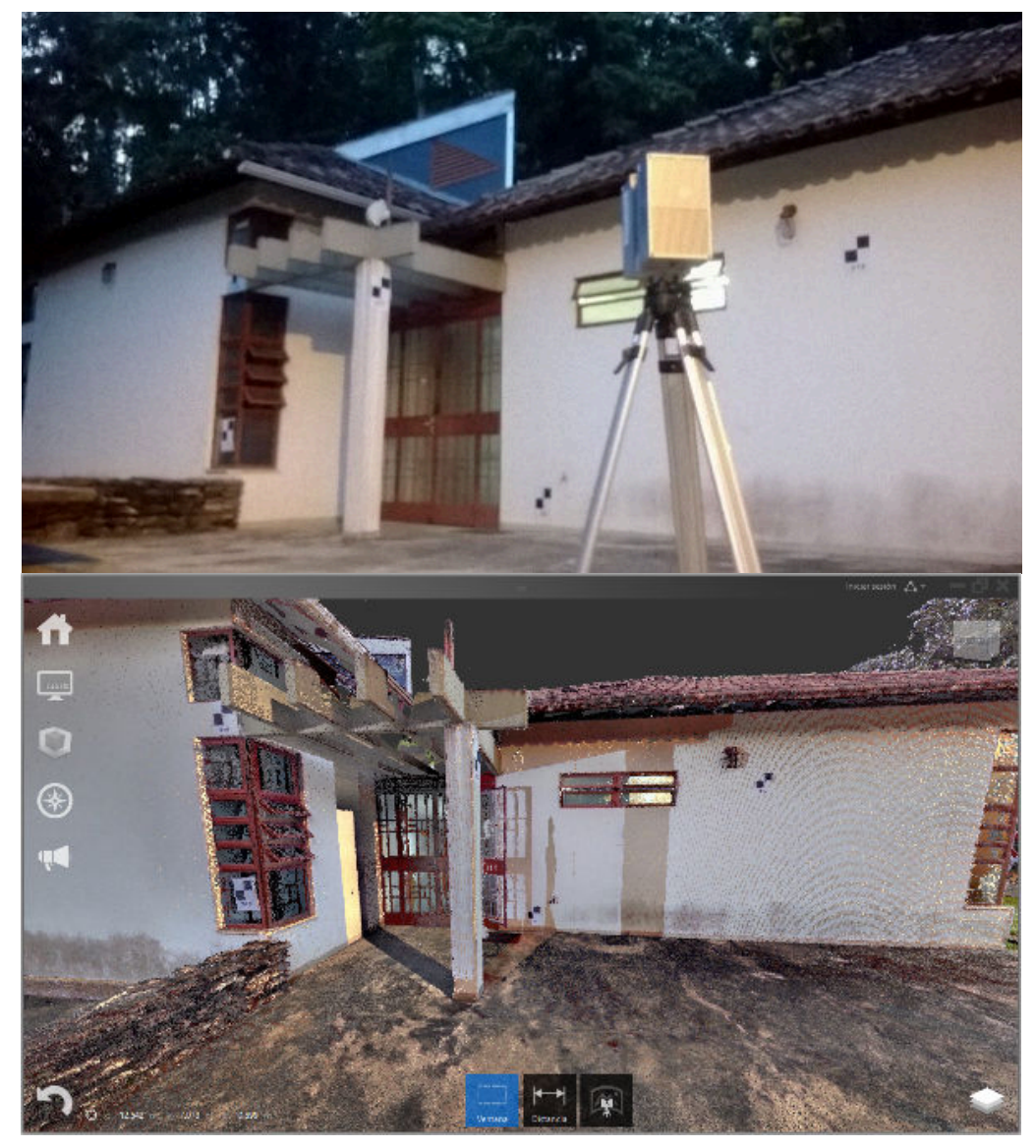

Fonte: resultados da pesquisa

\subsection{Fase 4 Processamento dos dados - Registro das cenas}

Como resultado do processo de escaneamento, foram geradas sete nuvens de pontos que, na fase de processamento dos dados, foram registradas (figura 4) em um único modelo de nuvem de pontos que pôde ser montado a partir do referenciamento pelos alvos inseridos na segunda fase do processo.

Nesta etapa foi utilizado o software Autodesk ReCap ${ }^{\circledR}$ lançado pela empresa com objetivo de executar este tipo de registro e visualizar arquivos de nuvens de pontos. A escolha por este software deve-se ao fato que o programa não exige grande performance computacional para registro das cenas.

Neste projeto, as sete nuvens demoraram duas horas de processamento resultando em um modelo unificado com aumentando a densidade de pontos devido à sobreposição dos arquivos. No software utilizado também é feita a omissão de pontos desnecessários para o modelo, ampliando a velocidade de visualização da cena (figura 5).

Figura 4: Integração de duas cenas (imagens) para unificação da 


\section{nuvem de pontos.}

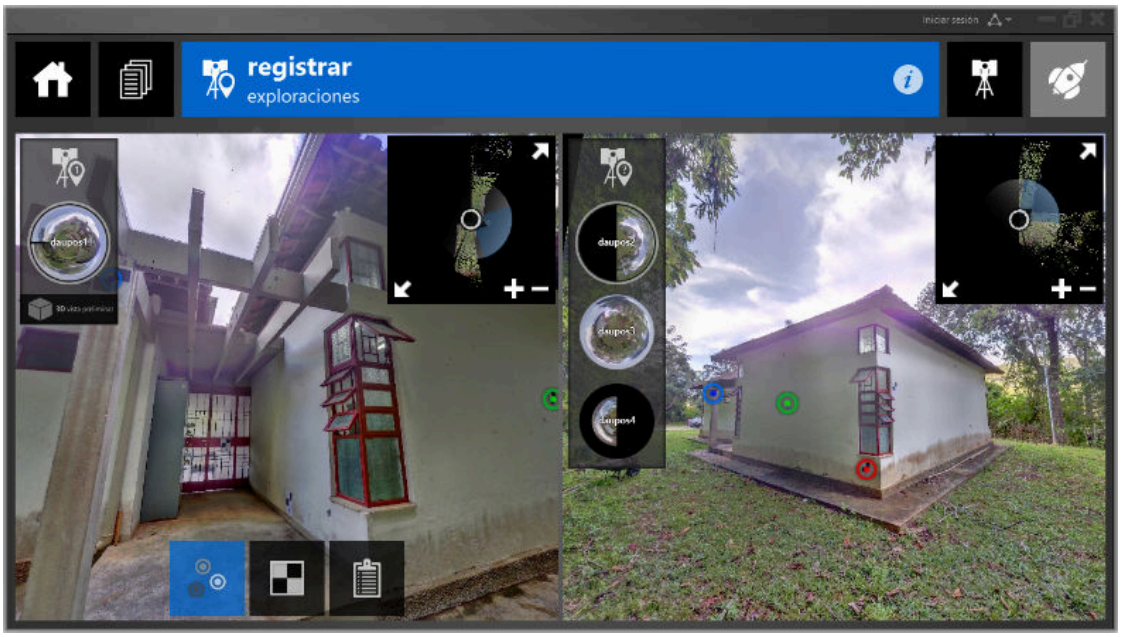

Fonte: resultados da pesquisa

Pfeifer e Briese (2007), lembram que apenas o feixe laser não é suficiente para uso em patrimônio, como pode ser exemplificado neste caso estudado onde somente o uso do laser atende à finalidade de cadastro do objeto escaneado devido à densidade de pontos, mas não é possível registro de cores, que dependem da integração da nuvem com as imagens geradas pela câmera interna do equipamento.

Figura 5: Visualização da nuvem de pontos unidas no software.

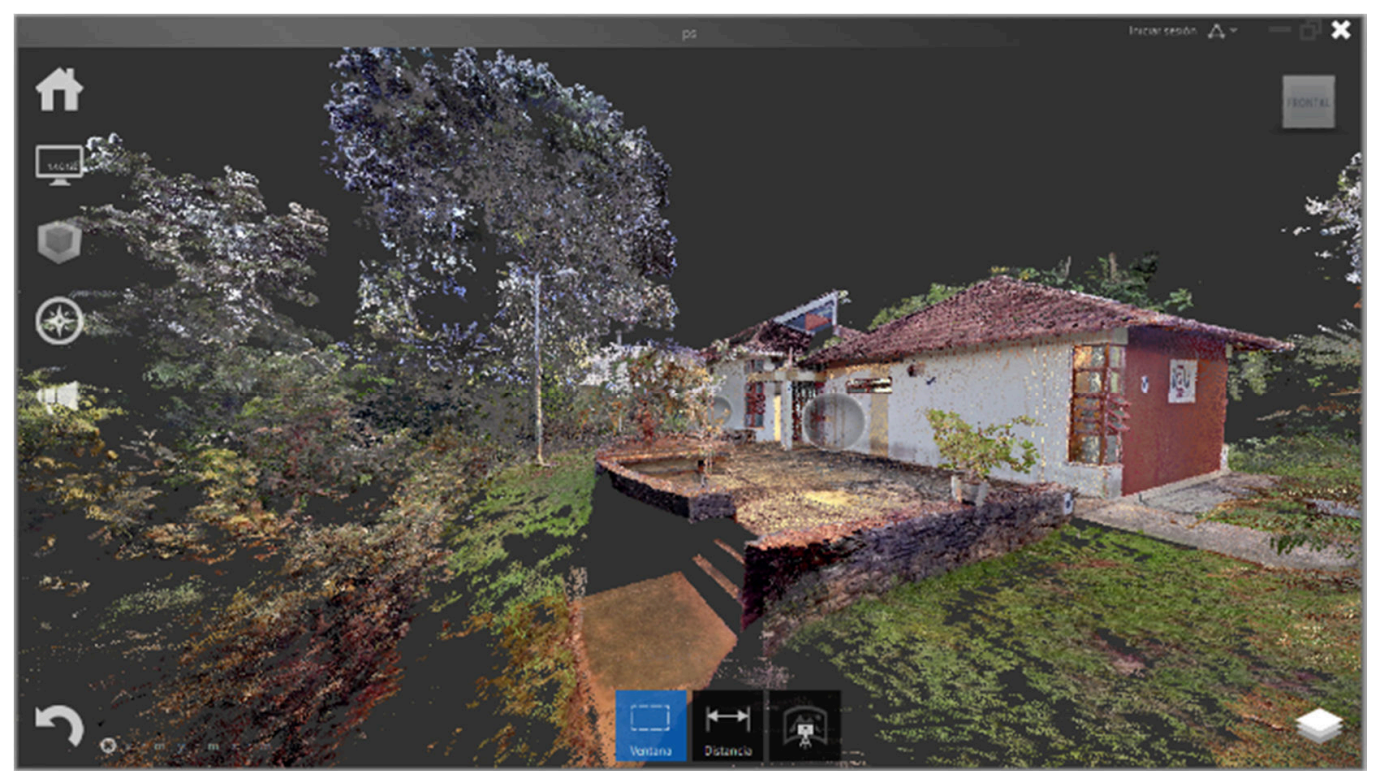

Fonte: resultados da pesquisa

\subsection{Modelagem tridimensional dos dados levantados}


Uma nuvem de pontos é um conjunto de elementos especiais pontuais apresentados espacialmente com coordenadas cartesianas que permitem tanto a aferição de medidas como a simulação dos ambientes internos e externos de uma edificação devido à densidade de pontos sugerindo a existência de superfícies. O estado atual da tecnologia LiDAR terrestre atingiu o patamar de definição de imagens que permite a representação de edificações e objetos sem a necessidade de desenvolvimento de modelos tridimensionais devido à densidade de pontos coletados.

Este avanço tecnológico habilita a divisão do processo de levantamento em duas etapas: a de cadastro e documentação, e a de modelagem para construção. Neste artigo, o cadastro da edificação através do laser foi a etapa inicial baseada no levantamento para registro cadastral e histórico, enquanto a segunda etapa explora a possibilidade de, a partir da mesma nuvem de pontos, gerar modelos simples com objetivo de extração de documentação, como plantas e cortes em um programa CAD, assim como a elaboração de modelos mais complexos com informação da construção em seu banco de dados.

A partir da unificação da nuvem de pontos em um único modelo de coordenadas, é possível sua exportação ou vinculação a outros arquivos de diferentes plataformas. Neste trabalho usamos o arquivo da nuvem unificada (.rcs) com os software Autodesk AutoCAD ${ }^{\circledR}$ e Revit ${ }^{\circledR}$.

O guia divulgado pela GSA (2009) esclarece que a modelagem nem sempre é a indicada porque, o processo de modelagem irá introduzir outros erros e a incerteza sobre o modelo é maior do que a dos dados puros. Desta forma o processo de modelagem só deve acontecer quando houver interesse em criação de um objeto composto por faces, o que pode ocorrer quando:

1. Não houver definição/resolução suficiente na nuvem, normalmente vinculada ao uso do equipamento;

2. Quando houver necessidade de criar um ambiente de simulação virtual que necessite aplicação de materiais e iluminação em modelos;

3. Prototipagem do objeto que determina a necessidade de redução da geometria ou preparação do material para o processo.

4. Quando a nuvem for usada como referência ou um as-built para modelagem sobre aquela situação.

Em situações diferentes das apresentadas o modelo de nuvem de pontos já contém dados suficientes para cadastro ou simulação do objeto mapeado, atingindo o estado de simular cores, detalhes e defeitos de objetos e elementos que compõe a superfície sem a necessidade de criação de superfície ou adição de fotos além das captadas pelo equipamento.

\subsubsection{Modelagem a partir de planta baixa documentada e integração com a nuvem de pontos}

O processo de modelagem no Autodesk AutoCAD ${ }^{\circledR}$, também segue uma série de passos para a geração de modelos e, neste caso, tomou como ponto de partida, um modelo 2D preexistente (figura 6), que foi resultante de em prévio levantamento da edificação a partir do qual se iniciou o processo de modelagem 3D. Este processo se deu a partir da extrusão de superfícies, tendo como resultado final um modelo de visualização tridimensional, sem materiais 
próprios da edificação, o que seria possível de se agregar em uma segunda etapa mediante a criação de materiais e sua inserção nas superfícies geradas.

Figura 6. Planta baixa e de cobertura em DWG.

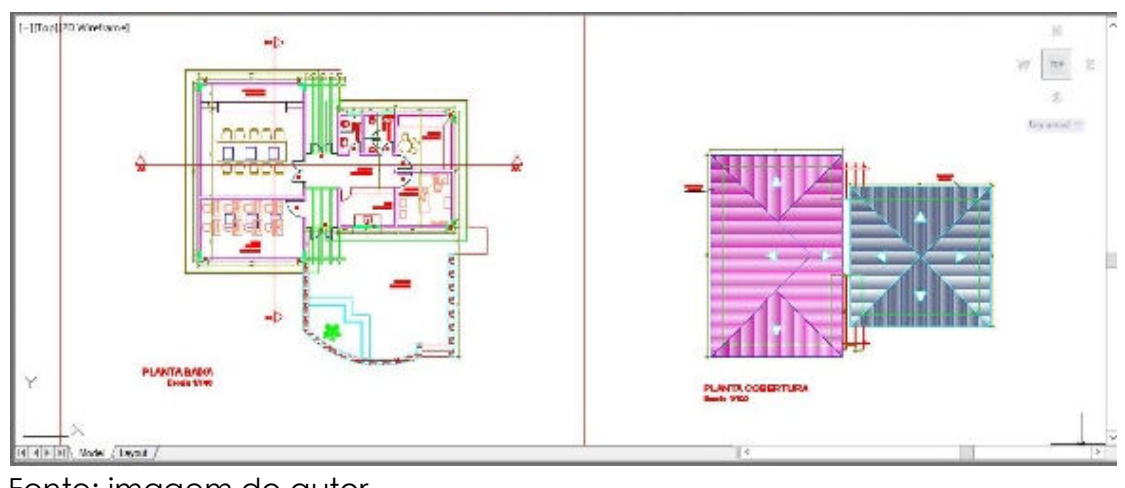

Fonte: imagem do autor

Assim que o modelo foi finalizado, a nuvem de pontos foi inserida no mesmo arquivo para aferição das medidas. De imediato foi verificada a ausência de elementos construtivos que não haviam sido coletados no momento da elaboração da planta baixa. Mesmo na revisão deste levantamento, não houve possibilidade de registro de parte do telhado por dificuldade de acesso. 0 resultado foi uma simplificação ou perda de informações sobre a edificação (figuras 7 e 8).

Figuras 7 -Inserção da nuvem de pontos (acima) no programa e visualização da incoerência quando relacionada com o modelo 3D.
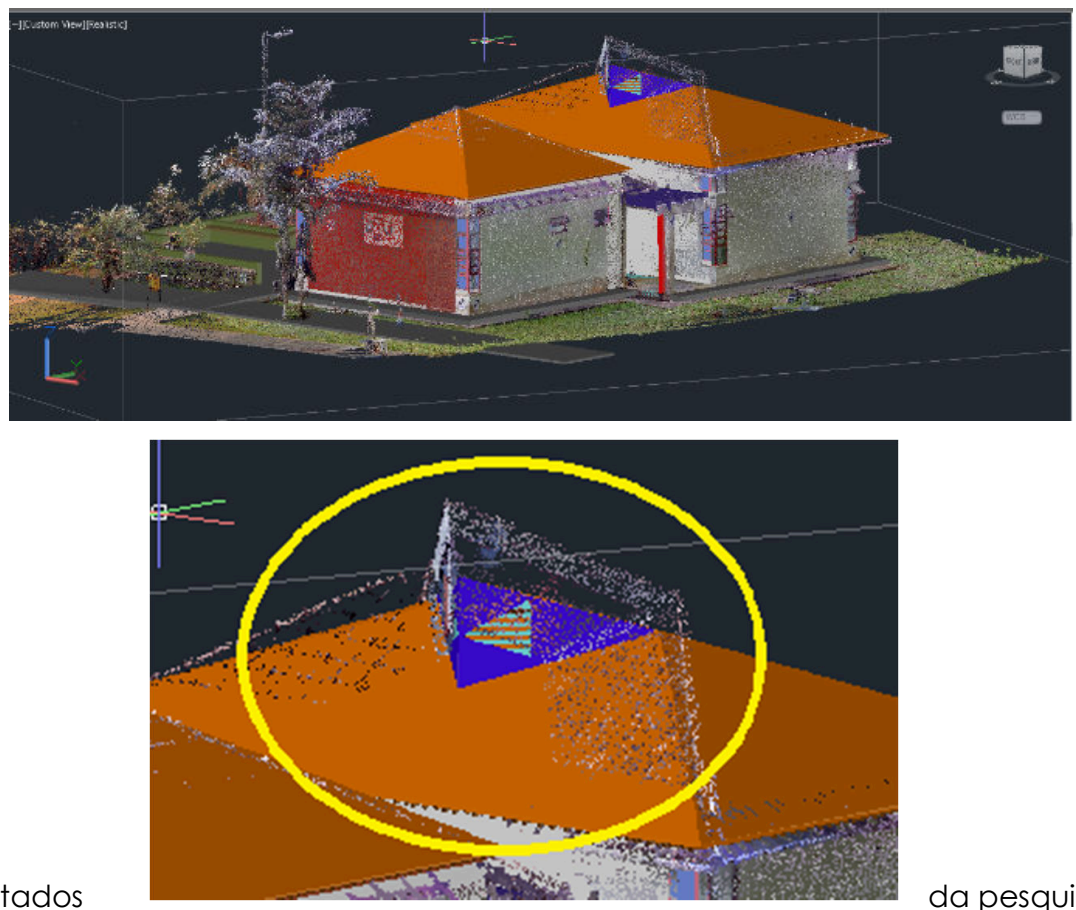
Figura 8: Nuvem de pontos, modelo 3D (.dwg) e incoerências visualizados no software.

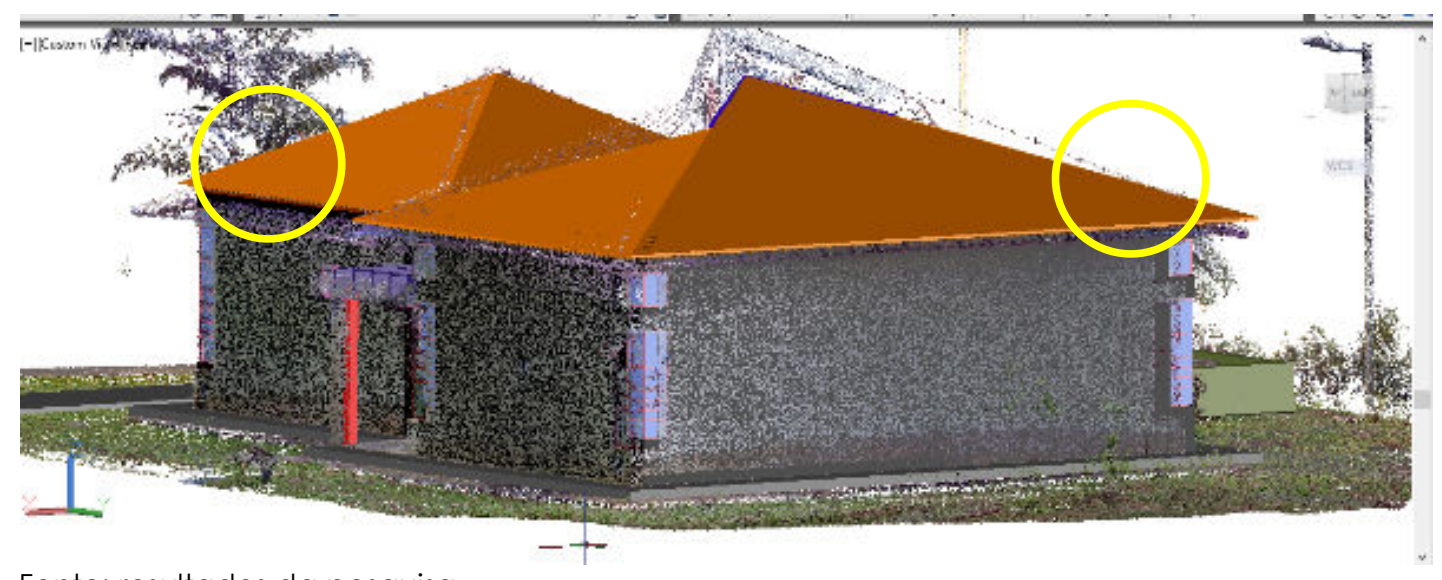

Fonte: resultados da pesquisa

Figura 9: Visualização da margem de erro entre modelo e nuvem.

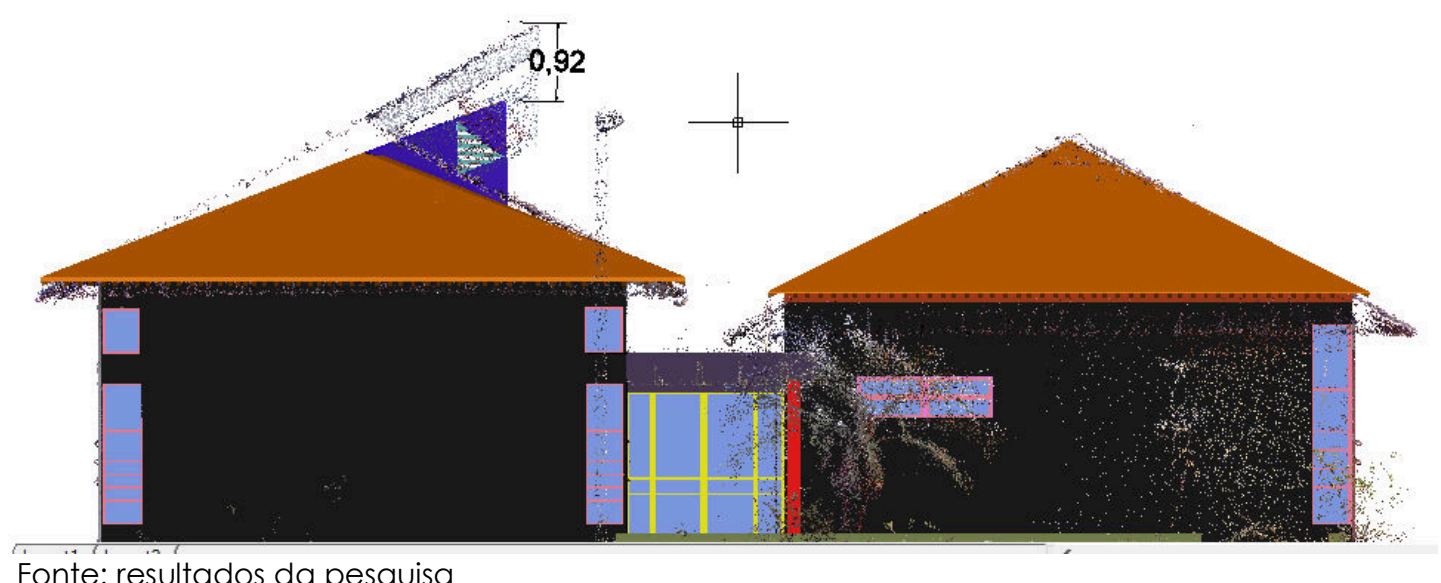

\subsubsection{Modelagem paramétrica a partir da nuvem de pontos}

Diferente do processo anterior de modelagem, que partiu de um conjunto de desenhos produzidos por outra equipe, o processo a seguir utilizou a nuvem mapeada como referência para a modelagem. Nesta ocasião o software elencado para modelagem foi o Autodesk Revit ${ }^{\circledR}$, porque permite o acréscimo de informações futuras para a geração de um modelo BIM, que, devido à interoperabilidade com $\circ$ Autodesk $\operatorname{ReCap}^{\circledR}$ usado para registro das cenas, reduzimos o risco de perda de informação e aceleramos o tempo de modelagem com a compatibilidade entre arquivos.

O processo de modelagem dentro de softwares CAD ou na plataforma BIM é baseado na importação da nuvem de pontos como uma referência externa e que não pode ser editada. Como o arquivo de nuvem de pontos nas resoluções mais altas possui milhões pontos, este processo de vinculo exige menos processamento de informação. 


\section{Imagem 10: Início do processo de modelagem do telhado no Autodesk Revit $^{\circledR}$.}

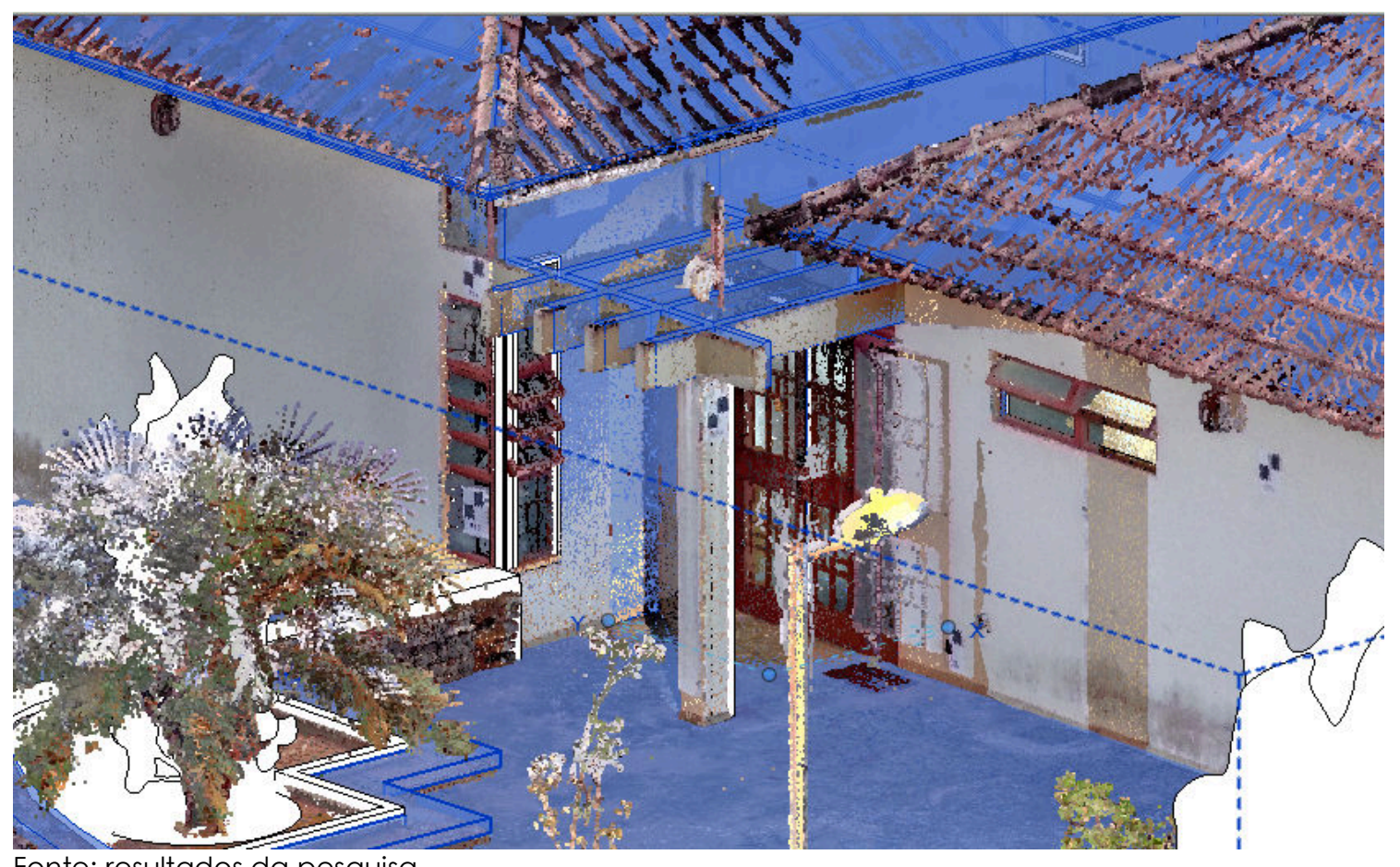

Fonte: resultados da pesquisa

Imagem 11: Modelo paramétrico sobre nuvem de pontos.

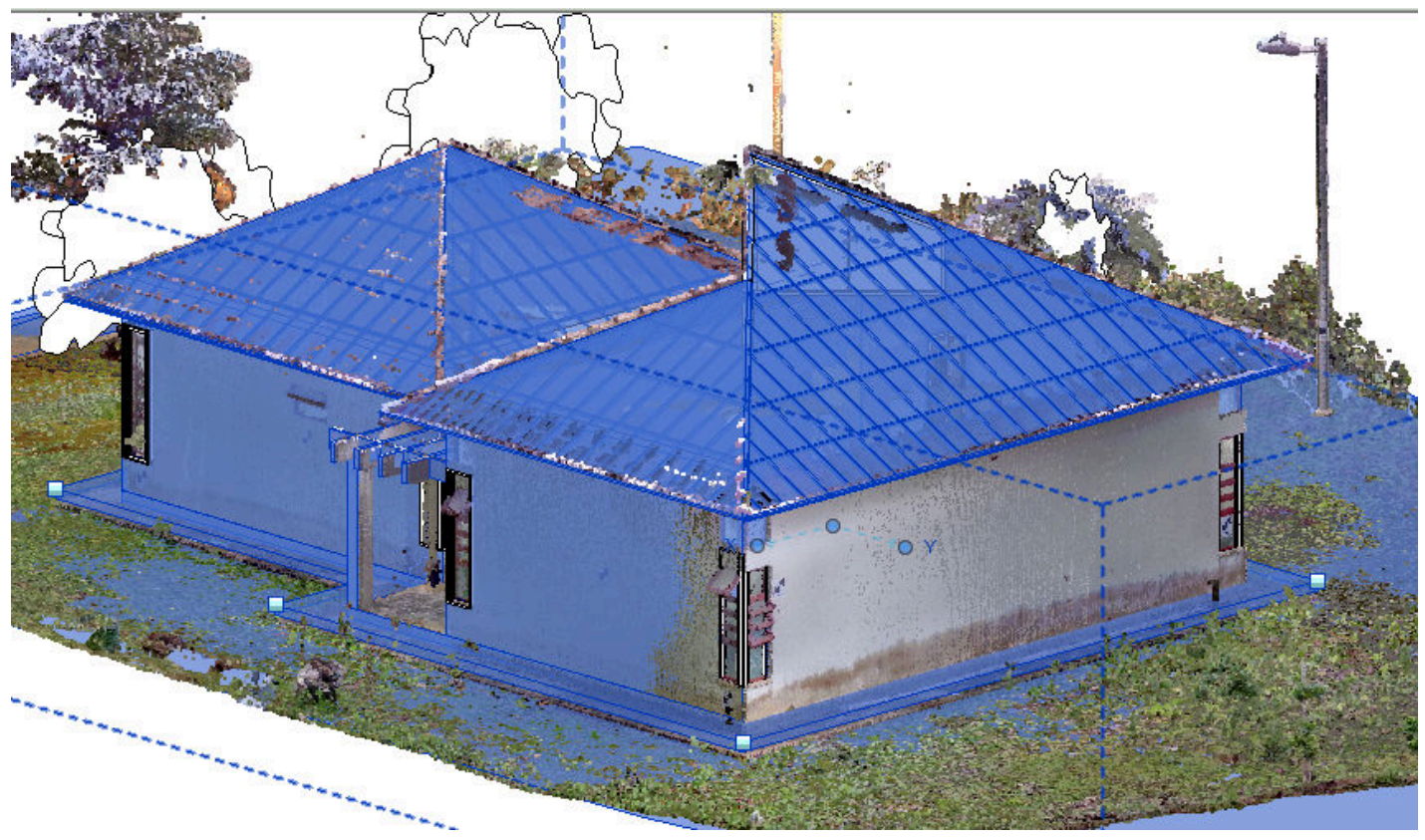

Fonte: resultados da pesquisa

A partir da inserção da nuvem como referência para modelagem, são apresentados alturas, comprimentos e inclinações de todos elementos 
construtivos que podem ser inseridos sobre a referência dos pontos da nuvem o que contribui para representação precisa da condição real.

Como o modelo gerado é paramétrico e orientado a objetos, sua modelagem consiste em representar elementos construtivos enquanto os desenhos e vistas do modelo são apresentadas automaticamente pelo programa. Este método de modelagem evita o retrabalho de ajustes de desenhos uma vez que uma alteração repercute em todos os documentos.

\section{CONSIDERAÇÕES FINAIS}

Todo este trabalho de modelagem sobre nuvem de pontos dependeu de plataformas diferentes (Autodesk AutoCAD, Autodesk Revit, Autodesk ReCap) e a interoperabilidade entre os software é necessária. O trânsito do arquivo deve ser evitado a fim de se evitar a perda de informação do mapeamento.

Um dos primeiros resultados e o mais evidente comparando a planta baixa obtida pelo levantamento tradicional e a condição real da edificação é a falta de concordância entre o desenho e a condição real, representado por diferenças de medidas superiores a $30 \mathrm{~cm}$ entre o mapeado em 2D e o modelo.

Outro ponto importante sobre este tipo de levantamento é que se baseia em produtos que simplificam a geometria da construção (cortes, plantas e fachada). Este processo de representação de uma condição da construção reduz a complexidade geométrica, cria redundância entre desenhos, ou seja, a mesma informação precisa ser representada em mais de uma planta ou corte, e esta situação habilita a ocorrência de contradições de desenho (em uma planta existe uma certa informação, mas no corte não foi existe ou possui medidas diferentes) e a modelagem a partir deste tipo de produto certamente irá operar por extrusão direta da planta ou corte, produzindo superfícies planas que são uma abstração e não representam os detalhes e irregularidades da superfície original.

Diferente de um levantamento gráfico por métodos convencionais, o levantamento laser opera sua exatidão de informações de acordo com configurações no equipamento, independente da escala na qual o objeto posteriormente. Diferente do processo convencional que admite imprecisão acima de $5 \mathrm{~cm}$ em desenhos na escala 1:100, o ambiente digital de visualização e modelagem permite utilizar em absoluto a informação coletada e, na modelagem paramétrica, a escolha da escala de representação sem perder informações originais.

Podemos também falar de eficiência que não se resume somente à qualidade, mas também a demanda de tempo para levantamento, já que, ao comparar a modelagem paramétrica que teve como suporte a nuvem de pontos com a modelagem tradicional que utilizou um levantamento anterior, determinou uma a diferença de vinte horas de modelagem gerada pela incerteza e incoerência dos dados usados no primeiro método.

Durante o processo de verificação do escaneamento da fachada, foi identificado que o laser ultrapassou a camada superficial de tinta que cobria a parede, revelando o logotipo da antiga função que a edificação abrigava. Este comportamento do feixe de luz ignorando camadas de tinta já foi verificado em 
alvos de papel usados experiências de campo e indica outra potente função para a tecnologia como levantamento arqueológico em sítios com edificações históricas revelando alterações realizadas nas edificações.

A precisão de informação obtida pelo escâner é visivelmente superior ao método tradicional, mas é dependente do processo de mapeamento que pode ampliar ou diminuir a margem de erro. De acordo com o fabricante do equipamento, a resolução utilizada neste estudo gera pontos distantes $6 \mathrm{~mm}$ uns dos outros (medido a 10m).

\section{REFERÊNCIAS}

GROETELAARS, N., AMORIN, A. Tecnologia 3D Laser Scanning: Características Processos e Ferramentas para Manipulação de Nuvens de Pontos. In: Anais do 15th Iberoamerican Congress of Digital Graphics. Santa Fe, Argentina. p. 490-494, nov. 2011.

U.S. General Services Administration. GSA Building Information Modeling Guide Series: 03 - GSA BIM Guide for 3D Imaging, 2009. Disponível em: <http://www.gsa. gov/graphics/pbs/GSA_BIM_Guide_Series_03.pdf>. Acesso em 10 abr. 2015.

Pfeifer, N., BRIESE,C. Laser scanning - principles and applications. In: Anais do 3rd International Exhibition and Scientific Congress - GeoSiberia, 2007. Novosibirsk, Russia, p. 93-112.

KERSTEN, T., STERNBERG, H., MECHELKE, K., Geometrical Building Inspection by Terrestrial Laser Scanning. In: FIG Working Week 2009: Surveyors Key Role in Accelerated Development. Eilat, Israel, 2009.

LICHTI, D. D., GORDON, S. J., STEWART, M. P. Ground-based laser scanners: operation, systems and applications: Geomatica. Vol. 56, no. 1, p. 21 - 33, 2002.

ALKAN, R. M., KARSIDAG, G. FIG Working Week 2012: Knowing to manage the territory, protect the environment, evaluate the cultural heritage. Rome, 2012. 